\title{
Domain III of Dengue Virus Serotype 2 Envelope: Expression at High Levels in Escherichia coli and Competitive Inhibition of Virus Entry
}

\author{
Yong- hong Yang ${ }^{1}$, Xue-li Zheng ${ }^{1}$, Lei-zi Qing ${ }^{1}$, Pin-ting Zhu ${ }^{1}$, Jing Pan ${ }^{1}$, Lei Luo ${ }^{2}$
}

Obejective The domain III of dengue virus type 2 envelope was cloned and expressed in Escherichia coli and the recombinant protein inhibited virus effect was tested.

Methods In this study, the domain III (DIII) protein of the dengue virus type-2 (DENV-2) envelope (E) antigen was expressed in Escherichia coli by fusion with a carrier protein. The protein was purified using enzymatic cleavage and affinity purification. Rabbit immunization and antibody detection was carried out. Inhibition of DENV-2 infection was observed by DENV-2 EDIII protein and its immunity rabbits serum.

Results The recombinant expression DENV-2 EDIII protein plasmid was constructed successfully. After isopropyl thiogalactoside induction, a specific soluble $29 \mathrm{kD}$ protein was obtained, and the expression product accounted for $68.87 \%$ of the total protein of the cell lysate. Western blotting demonstrated the reactivity of the recombinant protein with his-tag and DENV ( I - IV) monoclonal antibodies. The protein was purified using enzymatic cleavage and affinity purification. The purified recombinant EDIII protein inhibited the entry of DENV-2 into BHK-21 cells. DENV-2 plaque neutralization assays were carried out using serially diluted antibodies against EDIII protein. At a 1:16 dilution, the antibodies produced at least $90 \%$ neutralization of the DENV-2 virus. Furthermore, the antibodies continued to exhibit high neutralization effects (approximately $80 \%$ ) until the anti-EDIII antibody titer reached 1:1 024.

Conclusions DENV-2 EDIII was cloned and expressed successfully. DENV-2 EDIII protein could be useful in the development of inexpensive dengue vaccine. The data also suggested that DENV-2 employed an attachment molecule or receptor for its entry into $\mathrm{C} 6 / 36$ mosquito cells.

Key words: Dengue virus; Envelope domain III; Virus entry; Vaccine; Neutralizing antibodies

$\mathrm{D}$ engue virus is a mosquito-borne human pathogen that is a cause of serious public health concern worldwide, with approximately 100000000 cases of dengue infection and 500000 cases of hospitalizations due to dengue per annum. ${ }^{1}$ The fatality rate of the affected individuals is approximately $1 \%$ to $5 \%$, and it occurs mostly in children. ${ }^{1}$ However, the mechanisms involved in the pathogenesis of dengue hemorrhagic fever and dengue shock syndrome have not yet been elucidated. Currently, there are no approved human vaccines or therapeutic agents, and preventive

\footnotetext{
${ }^{1}$ Department of Etiobiology of School of Public Health and Tropical Medicine of South Medical University, No.1838, North Road Avenue, Guangzhou, Guangdong Province 510515, China

${ }^{2}$ Guangzhou Center for Disease Control and Prevention, No.1, Qide Road of Baiyun District, Guangzhou, Guangdong Province 510440, China

Correspondence: Xue-Li Zheng, Tel: +86-20-61648651, E-mail: zhengxueli2001@126.com
}

measures are largely focused on mosquito control. ${ }^{2-3}$

Dengue virus is a positive, single-strand RNA virus belonging to the flavivirus genus of the Flaviviridae family. It contains an $11 \mathrm{~kb}$ genome encoding three structural proteins (capsid, premembrane, and envelope [E]) and seven nonstructural proteins (NS1, NS2A, NS2B, NS3, NS4A, NS4B, and NS5). ${ }^{3}$ The E glycosylated protein, which is the major structural protein presented on the surface of the mature dengue virions, is a type I integral membrane protein. This protein consists of three $\beta$-barrel domains. Domain I (D I ) contains the N-terminus but is centrally located in the molecule. Domain II (D II) is elongated and it mediates the dimerization of $\mathrm{E}$. It also contains the hydrophobic and well-conserved fusion peptide at its distal end. Domain III (DIII) is an immunoglobulin (Ig)-like domain, which has 
been predicted to be involved in receptor binding and antibody neutralization. ${ }^{4}$ In addition, several studies have demonstrated that both recombinant DIII proteins and antibodies generated against EDIII of genus flavivirus can inhibit the entry of the virus into target cells. ${ }^{5-7}$ Recently, the EDIII protein was demonstrated to be critical for vaccine development. ${ }^{8-13}$ The most effective way of preventing viral infection is by inhibiting the entry of the virus particles into target cells. In this study, we aimed to investigate the antigenic and antagonistic properties of the EDIII protein of dengue virus serotype 2 (DENV-2). The EDIII was expressed in Escherichia coli and purified. The results of our study have important implications in the design of an antiviral agent or a subunit vaccine targeting dengue virus infection.

\section{MATERIALS AND METHODS}

\section{Cell lines and viruses}

DENV-2 strain New Guinea C was used in this study. Baby hamster kidney (BHK) cells were cultured in Glasgow minimum essential medium (GMEM) supplemented with $5 \%$ fetal bovine serum (FBS), $10 \%$ tryptose phosphate broth, and $20 \mathrm{mM} \mathrm{N}-2$ hydroxyethylpiperazine-N-2-ethane sulfonic acid (HEPES) buffer. Mosquito cells derived from the Aedes albopictus $\mathrm{C} 6 / 36$ clone were cultured at $28^{\circ} \mathrm{C}$ in RPMI medium with $10 \%$ FBS. All the media were supplemented with $100 \mu \mathrm{g} / \mathrm{ml}$ of L-glutamine and 100 $\mu \mathrm{g} / \mathrm{ml}$ of penicillin-streptomycin. Throughout this study, DENV-2 was propagated on C6/36 cell lines.

\section{Cloning and expression of recombinant DENV-2 DIII protein}

Preparation of DENV- $2 E$ gene domain III coding sequence

Total RNA was prepared from DENV-infected C6/36 cell lines that had been inoculated with live DENV-2. The total RNA was then reverse transcribed using random hexamers and superScript ${ }^{\mathrm{TM}}$ (Invitrogen). The E protein DNA fragment was amplified by RTPCR and ligated into pMD-18-T to construct pMD18-T-DENV-2-E. Viral genomic RNA was used as a template to obtain the E gene fragment DNA by RT-PCR, which was carried out with the following parameters: $94^{\circ} \mathrm{C}$ for $10 \mathrm{~min}, 35$ cycles of $94^{\circ} \mathrm{C}$ for 90 s, $56.9^{\circ} \mathrm{C}$ for $100 \mathrm{~s}$, and $72^{\circ} \mathrm{C}$ for $100 \mathrm{~s}$; followed by a final extension step at $72^{\circ} \mathrm{C}$ for $15 \mathrm{~min}$. The sense strand primer was DENV-2-E-F (5' -GGGCCATATG
TCAATGACAATGCGTT-3' , where the underlined sequence represents the $N d e$ I restriction site) and the antisense strand primer was DENV-2-E-R (5' -AATTCTCGAGATCGGCCTGCACCAT-3' , where the underlined sequence represents the Xho I restriction site). The gene sequences of the $\mathrm{C}$-terminal EDIII were amplified by PCR using primers DENV2-EDIII-F (5' -CGCGGATCCGGAATGCATACT CTATGTGCA-3' ${ }^{\prime}$, where the underlined sequence represents the $B a m \mathrm{H}$ I restriction site) and DENV2-EDIII-R (5' -TCTCGAAGCTTTTATTTCTTCT TAAACCAGTTGAGCT- $3^{\prime}$, where the underlined sequence represents the Hind III restriction site), with the following parameters: $98^{\circ} \mathrm{C}$ for $5 \mathrm{~min} ; 30$ cycles of $95^{\circ} \mathrm{C}$ for $30 \mathrm{~s}, 59.6^{\circ} \mathrm{C}$ for $60 \mathrm{~s}$, and $72^{\circ} \mathrm{C}$ for $45 \mathrm{~s}$; with a final extension step of $72^{\circ} \mathrm{C}$ for $10 \mathrm{~min}$. A 320 bp region of the DENV-2E gene domain III sequence flanked by the Bam H I and Hind III restriction sites was obtained after PCR.

\section{Construction of the recombinant plasmid}

The PCR product was purified with a Qiaquick DNA purification kit (Qiagen) and digested with Bam H I and Hind III, then ligated into the corresponding BamH I and Xho I restriction sites in the expression vector pET32a $(+)$ (Novagen, Darmstadt, Germany) to generate the recombinant plasmid pET32a-DENV2-EDIII. The resulting plasmids expressed the EDIII proteins together with a thioredoxin tag for affinity purification at the $\mathrm{N}$-terminus. An enterokinase cleavage site is also present in the DENV-2 EDIII gene. The nucleotide sequences were confirmed with DNA sequence analysis.

\section{Expression and purification of recombinant DENV-2 EDIII protein}

Cells of the E. coli BL21 strain (DE3) with the pET32a vector containing EDIII were cultured until an $A$ of 0.6 was reached at $600 \mathrm{~nm}$. Protein expression was subsequently induced by adding isopropyl thiogalactoside (IPTG) to achieve a final concentration of $1 \mathrm{mM}$, followed by incubation at $25^{\circ} \mathrm{C}$ for $5 \mathrm{~h}$. The cells were then harvested by centrifuging at $9000 \times \mathrm{g}$ for $15 \mathrm{~min}$ at $4^{\circ} \mathrm{C}$. The pellets were thawed on ice and resuspended in $50 \mathrm{mM}$ Tris- $\mathrm{HCl}(\mathrm{pH} 7.0)$ containing $500 \mathrm{mM} \mathrm{NaCl}$. The resuspended cells were lysed by sonication on ice for $20 \mathrm{~s}$; this process was repeated four times. After centrifugation, the supernatant was collected, and the pellet containing the inclusion bodies was resuspended with buffer B [20 mM Tris- $\mathrm{HCl}(\mathrm{pH} 8.0) / 5 \mathrm{mM}$ EDTA/100 NaCl]. 
In order to determine whether the recombinant protein is soluble, aliquots of the supernatant and the pellet were analyzed by sodium dodecyl sulfatepolyacrylamide gel electrophoresis (SDS-PAGE). The supernatant was subjected to affinity purification using a Profinity ${ }^{\mathrm{TM}}$ IMAC Ni-charged resin column (Bio-Rad), and fusion proteins were eluted from the column. The immunoreactivity of the recombinant protein was analyzed by Western blotting using histag monoclonal antibodies (mAbs) and anti-DENV (1-4) mAbs. The respective recombinant proteins were cleaved with enterokinase and dialyzed against phosphate-buffered saline (PBS) overnight at $4^{\circ} \mathrm{C}$.

\section{Rabbit immunization and antibody detection}

Two groups of adult New Zealand rabbits $(2-3 \mathrm{~kg})$ were used for this experiment. Two rabbits of the first group were intraperitoneally injected on days 0,10 , 24, and 38 with $400 \mu \mathrm{g}$ purified EDIII protein in 1 ml PBS emulsified with $0.5 \mathrm{ml}$ Freund' s adjuvant. The second group of rabbits (only one) served as the negative control and received PBS without protein for 10 days. After the last injection, the rabbits were bled, and their sera were collected and stored at $-70^{\circ} \mathrm{C}$ until analysis.

The anti-DENV-2 EDIII antibody titers in the rabbit sera were evaluated by an enzyme-linked immunosorbent assay (ELISA) kit. The wells of a 96-well microplate were coated with purified DENV-2 EDIII protein $(100 \mu \mathrm{g}$ in $100 \mu \mathrm{l} /$ well $)$ by incubation at $37^{\circ} \mathrm{C}$ for $2 \mathrm{~h}$; the protein was blocked by addition of $1 \%$ bovine serum albumin (BSA) in PBS-T followed by incubation for $2 \mathrm{~h}$ at $37^{\circ} \mathrm{C}$. The wells were washed three times, incubated with peroxidaseconjugated goat anti-rabbit IgG (1:5 000 in blocking buffer; Southern Biotech, Birmingham, AL, USA) for $30 \mathrm{~min}$ at $37^{\circ} \mathrm{C}$. After washing thrice, $100 \mu 1$ $3,3^{\prime}, 5,5^{\prime}$-tetramethylbenzidine was added to each well, followed by incubation for $15 \mathrm{~min}$ at $37^{\circ} \mathrm{C}$. The reaction was stopped by adding $50 \mu \mathrm{l}$ of $1 \mathrm{M} \mathrm{H}_{2} \mathrm{SO}_{4}$, and the absorbance was read at $450 \mathrm{~nm}$. The sera were tested by Western blot analysis for the presence and specificity of antibodies against DENV-2 EDIII protein.

Inhibition of DENV-2 infection by soluble DENV2EDIII protein

Soluble EDIII protein and BSA (at concentrations of 10,25 , and $100 \mu \mathrm{g} / \mathrm{ml}$ ) were incubated with BHK-21 $\left(1 \times 10^{6}\right.$ cells $)$ in a cell culture medium for $1 \mathrm{~h}$ at $4{ }^{\circ} \mathrm{C}$. Unbound protein molecules were removed by washing the cells three times with PBS. Thereafter, the cells were infected with DENV-2 (at a multiplicity of infection [MOI] of 10) and incubated for $1 \mathrm{~h}$ at $37^{\circ} \mathrm{C}$. During the inoculation period, excess or unbound virus was inactivated with acid citrate buffer ( $\mathrm{pH} 2.8$ ) and removed by washing three times with PBS. This was followed by the addition of the respective maintenance medium (DMEM or L15 with $2 \%$ fetal calf serum [FCS]) and incubation at $28^{\circ} \mathrm{C}$ or $37^{\circ} \mathrm{C}$ for 3 days before the virus was harvested for plaque assays. Three independent experiments were performed.

\section{DENV-2 plaque neutralization assay}

Purified rabbit polyclonal antibodies generated against soluble EDIII protein or pre-immunized sera (control) were evaluated using plaque reduction assay to determine the ability of these antibodies to neutralize DENV-2 infectivity. We prepared $50 \mu \mathrm{g}$ of $500 \mu \mathrm{g} /$ $\mathrm{ml}$ antibodies in two-fold serial dilutions (from 1:2 to 1:4 096) in microcentrifuge tubes. The DENV-2 titer was adjusted to plaque-forming units (pfu) in $50 \mu 1$ of virus diluent ( $10 \%$ concentrated Hank' s balanced salt solution and $0.1 \% \mathrm{BSA}$; $\mathrm{pH} 7.2-7.4$ ), and added to the microcentrifuge tubes containing the antibodies at different serial dilutions. The antibody and virus were mixed by pulse centrifugation, and the mixture was incubated for $2 \mathrm{~h}$ at $37^{\circ} \mathrm{C}$. A 24-well plate with confluent monolayers of $\mathrm{C} 6 / 36$ cells was rinsed once with virus diluent, and $100 \mu \mathrm{l}$ of the antibody-virus mixture was added to the appropriate wells. The plate was incubated at $37^{\circ} \mathrm{C}$ for $1 \mathrm{~h}$. The excess inocula were removed by rinsing the cell monolayers with PBS. Then, overlay medium ( $2 \%$ carboxymethyl cellulose with RPMI-1640 medium containing 2\% FCS) was added to each well, and the plates were further incubated for 5 days at $37^{\circ} \mathrm{C}$ with $5 \% \mathrm{CO}_{2}$. The virus plaques were stained with $0.5 \%$ crystal violet.

\section{RESULTS}

\section{Cloning and expression of DENV-2 EDIII}

The $1.5 \mathrm{kD}$ full-length E protein gene fragment of DENV-2 was obtained by RT-PCR amplification. The amplified $\mathrm{E}$ fragment was digested with Nde I and Xho I ligated into the pMD-18-T vector to obtain the construct pMD-18-T-DENV-2E. Sequencing analysis of the amplified $\mathrm{E}$ fragment revealed no mutations. 


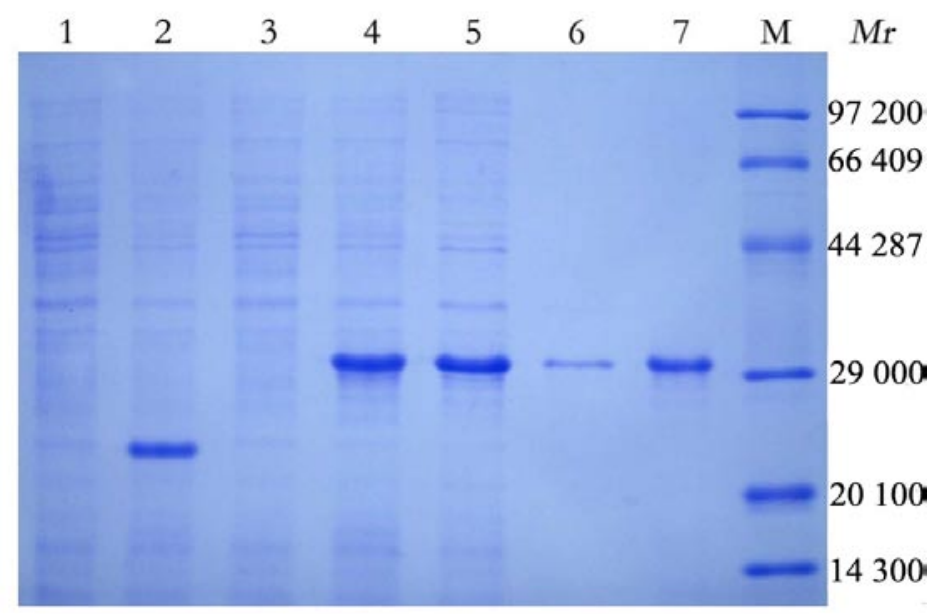

Figure 1. The expression of recombinant DENV-2 EDIII protein.

Notes: Total protein from bacteria transformed with pET-32a-DENV-2-EDIII or pET-32a(+) were separated by $12 \%$ SDS-PAGE and stained with Coomassie blue. Lanes: 1. BL21(DE3)/pET-32a(+) before induction; 2. BL21(DE3)/pET-32a(+) after induction; 3. BL21(DE3)/pET-32a(+)DENV-2-EDIII before induction; 4. BL21(DE3)/pET-32a(+)-DENV-2-EDIII after induction; 5. Supernatant of BL21(DE3)/pET-32a(+)-DENV2-EDIII after sonication; 6. Pellet of BL21(DE3)/pET-32a(+)-DENV-2-EDIII after sonication; 7. Purified recombinant protein with Ni-NTA hisbound resin; M, Molecular mass markers. The arrow indicates the WNV-DIII protein.

A

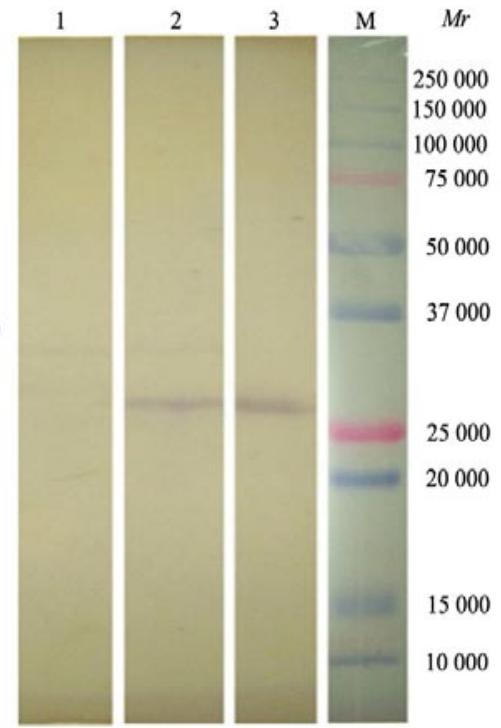

B

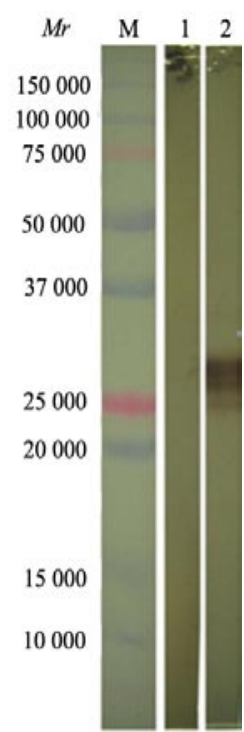

A

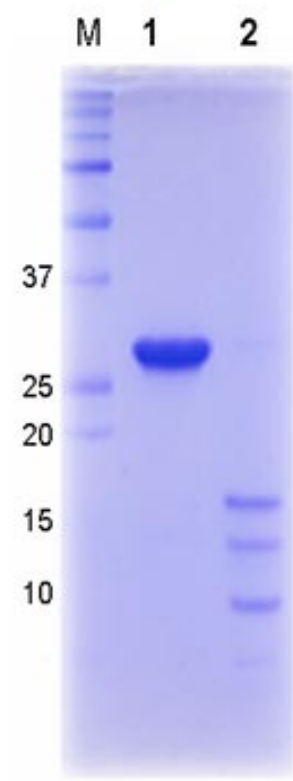

B

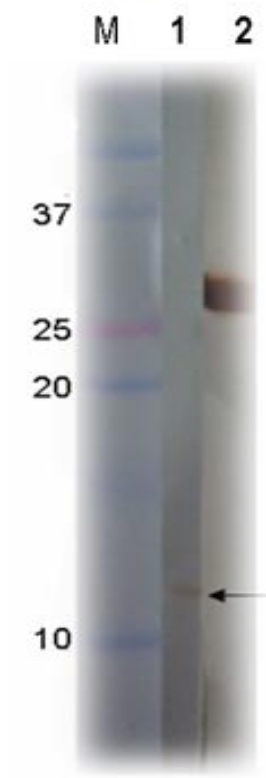

Figure 2. Analysis of recombinant protein using Western blot. Notes: (A) Analysis of recombinant protein with his-tag monoclonal antibodies using Western blot. Total protein from bacteria transformed with pET-32a(+)-DENV2-EDIII were separated by $12 \%$ SDSPAGE, transferred to a nitrocellulose membrane, and subjected to Western blot analysis using his-tag monoclonal antibodies (mAbs). Lanes: 1. BL21(DE3)/pET-32a(+)-DENV2-EDIIIbefore induction; 2. BL21(DE3)/pET-32a(+)-DENV-2-EDIII after induction; 3. Purified recombinant protein; M: Prestained protein marker. (B) Analysis of recombinant protein with DENV monoclonal antibodies using Western blot analysis. M: Prestained protein marker; Lanes: 1, BL21(DE3)/ pET-32a(+)-DENV-2-EDIII before induction; 2, BL21(DE3)/pET32a(+)-DENV-2-EDIII after induction.

Figure 3. Expression of recombinant DENV-2 EDIII envelope proteins.

Notes: The DENV-2 EDIII proteins were separated on 12\% SDSPAGE and stained with Coomassie blue. Recombinant DENV-2 EDIII envelope proteins were obtained after enterokinase cleavage and purification. M: Protein marker; Lanes: 1. Purified recombinant protein with Ni-NTA resin; 2. Cleavage with enterokinase to remove the thioredoxin and his tags of the recombinant protein. (B) Analysis of cleavage with enterokinase to remove the thioredoxin and his tags of the recombinant protein with DENV-2 monoclonal antibodies using Western blot. Lanes: 1. Purified recombinant protein; 2. Cleavage with enterokinase to remove the thioredoxin and his tags of recombinant protein; M: Protein marker. 


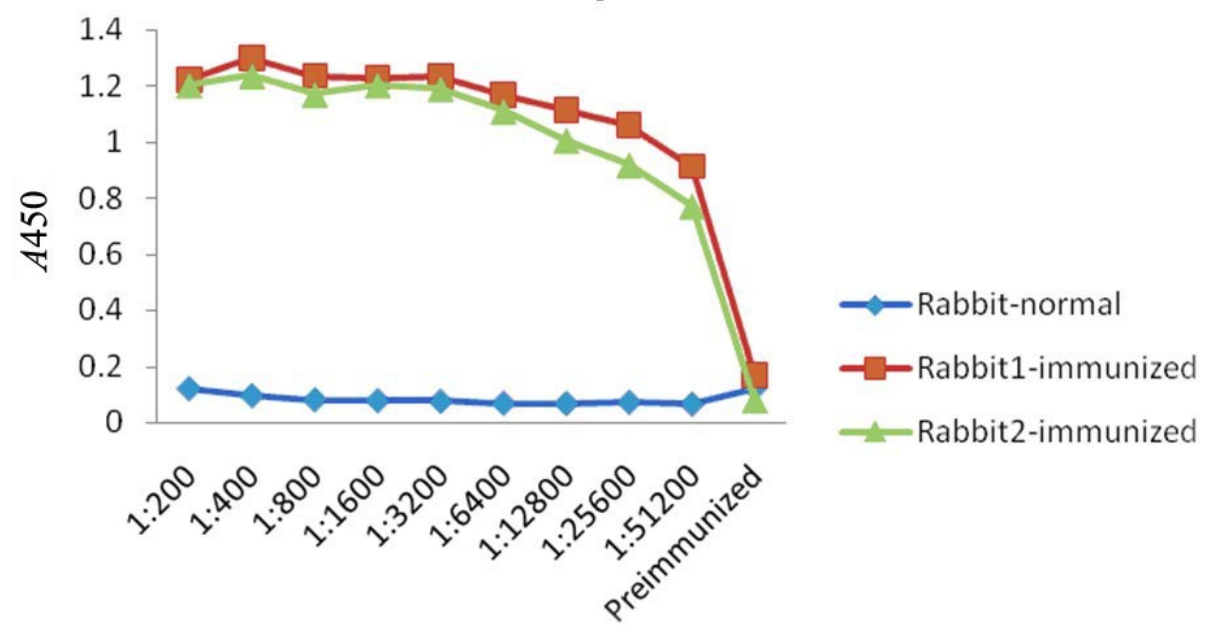

Figure 4. Detection of antibody titers in sera from immunized rabbits.

Notes: Serial two-fold dilutions of sera from rabbits immunized with DENV-2 EDIII protein (red rectangles and green triangles) or control group (blue diamonds) were detected by ELISA using DENV-2 EDIII protein as a capture antigen.

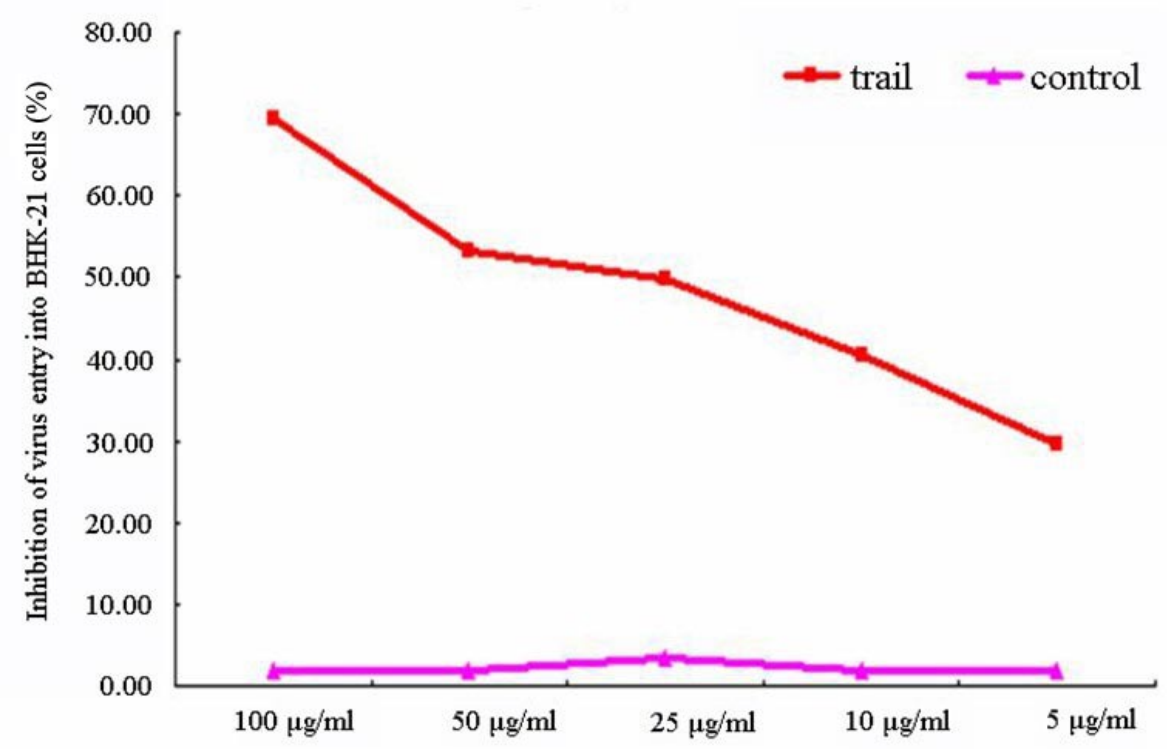

Figure 5. Competitive inhibition of DENV-2 entry into Vero cells with soluble recombinant DENV-2 EDIII protein.

Notes: Vero cells were first incubated with different concentrations of soluble DENV-2 EDIII protein or BSA (negative control). Entry of DENV-2 is significantly blocked in the presence of DENV-2 EDIII protein.

The 320 bp DENV-2 EDIII protein was obtained by using the pMD-18-T-DENV-2E construct as a template. The amplified DENV-2 EDIII fragment was digested with Bam H I and Hind III and ligated into the expression vector $\mathrm{pET} 32 \mathrm{a}(+)$ to obtain the construct pET32a(+)-DENV-2-EDIII. This construct was sequenced and compared to the NGC sequence. Both sequences exhibited $100 \%$ homology. The recombinant pET32a(+)-DENV-2-EDIII plasmid was transformed into BL21(DE3) host cells and induced by IPTG for $5 \mathrm{~h}$. Total protein was obtained and separated by $12 \%$ SDS-PAGE. The recombinant DENV-2 EDIII protein was expressed as a fusion protein with thioredoxin tag. A $29 \mathrm{kD}$ protein was detected in the protein extract obtained from E. coli containing the pET32a- DENV-2-EDIII plasmid, but this protein was not present in the extract obtained from cultures transformed with pET32a. Recombinant protein accounted for $68.87 \%$ of the recombinant $E$. coli protein. This recombinant protein was purified by Ni-NTA resin affinity chromatography and his-bind kits, and the obtained protein had a purity of $77.68 \%$. 


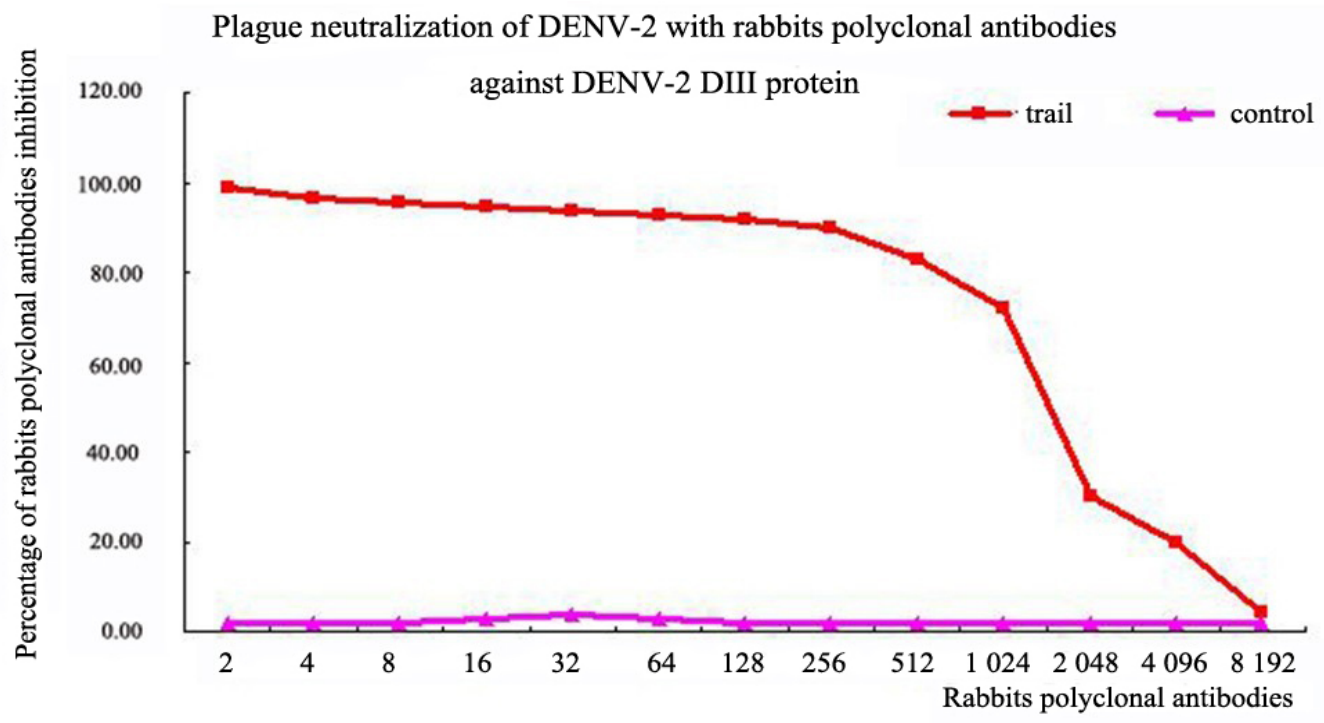

Figure 6. Plaque neutralization of DENV-2 with rabbit polyclonal antibodies to DENV-2 EDIIIprotein.

Notes: The polyclonal antibodies against DENV-2 EDIII protein were diluted in a two-fold series. Equal volumes (50 $\mu$ l) of anti-DENV-2 EDIII protein antibodies and DENV-2 (500 pfu) were incubated for $1 \mathrm{~h}$ before this mixture was overlaid on a C6/36 cell monolayer. Plaques were stained with $0.5 \%$ crystal violet. Virus diluent without antibodies was used as the negative control. Over $90 \%$ of the DENV-2 was neutralized when the antiserum was used at a dilution of up to 1:256. The neutralization capacity was maintained at $80 \%$ at dilutions of up to $1: 1024$. The percentage neutralization decreased exponentially thereafter, and antiserum dilution of 1:8 192 produced $0 \%$ neutralization.

Western blotting assay was performed using histag mAbs and DENV (1-4), and the results revealed that the $29 \mathrm{kD}$ protein was the recombinant EDIII protein (Figure 2). The thioredoxin and his tags were removed by cleavage with enterokinase, and extensive dialysis in PBS was performed to obtain the EDIII protein. The soluble EDIII protein was found to be approximately $12 \mathrm{kD}$ (Figure 3).

\section{Recombinant bacteria induced optimization of expression conditions}

The recombinant bacteria were induced by incubating with $1 \mathrm{mM}$ IPTG for $37^{\circ} \mathrm{C}$ and $25^{\circ} \mathrm{C}$ for $5 \mathrm{~h}$. The cells were then collected, resuspended in lysis buffer, and sonicated, after which they were analyzed by SDSPAGE. As shown in Figure 1, the expression of the recombinant protein, which was detected in soluble form in the supernatant, was higher at $25^{\circ} \mathrm{C}$ than at $37^{\circ} \mathrm{C}$; therefore, $25^{\circ} \mathrm{C}$ was chosen as the optimum temperature for induction. Recombinant bacteria showed optimum growth and protein expression at $25^{\circ} \mathrm{C}$. Induction times of $2,3,4,5$, and $6 \mathrm{~h}$ were chosen to identify the optimum induction time. The cells were collected and analyzed by SDS-PAGE. Results revealed that expression of the recombinant protein gradually increased with longer induction time, after which the protein expression gradually reached a plateau; thus, the optimum time to obtain high protein expression was deemed to be $6 \mathrm{~h}$. The induced concentration of the optimization was chosen. Finally, 0.1, 0.2, 0.4, 0.6, 0.8, and $1 \mathrm{mM}$ IPTG was added to the bacteria to determine the concentration of IPTG that yielded optimum protein expression, and the mixture was induced by incubation at $25^{\circ} \mathrm{C}$ for $6 \mathrm{~h}$. The cells were then harvested and analyzed by $12 \%$ SDS-PAGE. The recombinant protein expression gradually increased with the increasing concentrations of IPTG, and stabilized at $0.8 \mathrm{mM}$ IPTG, without further significant increase in expression. Therefore, 1 $\mathrm{mM}$ was chosen as the best concentration of IPTG for the induction of recombinant protein expression (data not shown).

\section{Competitive inhibition of DENV-2 by soluble EDIII proteins}

Soluble recombinant EDIII proteins were used to produce antibodies against the EDIII protein in New Zealand rabbits. Sera were tested by Western blotting to detect the presence and specificity of the generated antibodies against the EDIII proteins (Figure 4). Preimmune sera did not contain antibodies to detect the presence of DENV-2 in Western blot analysis.

Since EDIII has been proposed to be a receptorbinding domain, we investigated the ability of the recombinant EDIII to bind to cells and block the entry of DENV-2. To this end, BHK-21 cells were first 
incubated with various concentrations $(25-100 \mu \mathrm{g} /$ $\mathrm{ml}$ ) of soluble recombinant EDIII protein or BSA (as the control) at $4^{\circ} \mathrm{C}$ for $1 \mathrm{~h}$. DENV-2 was added to the pretreated cells and the virus entry was quantified.

It was revealed in Figure 5 that pretreatment of BHK-21 cells with EDIII protein dose-dependently inhibited DENV-2 entry, and pretreatment with 100 $\mu \mathrm{g} / \mathrm{ml}$ protein resulted in greater than $60 \%$ inhibition of DENV-2 entry. Different concentrations of BSA were used as the background control, and no significant inhibition occurred when BSA was used (data not shown).

\section{Rabbit polyclonal antibodies neutralized recombinant EDIII protein}

To further confirm that the EDIII protein is the receptor-binding domain, rabbit polyclonal antibodies against recombinant EDIII proteins were produced and used in a DENV-2 plaque neutralization assay. The rabbit polyclonal antibodies were confirmed by Western blotting to specifically bind to EDIII protein. The DENV-2 plaque neutralization assays were carried out using serially diluted antibodies against EDIII protein. At a 1:16 dilution, the antibodies produced at least $90 \%$ neutralization of the DENV-2 virus. Furthermore, the antibodies continued to exhibit high neutralization effects (approximately 80\%) until the anti-EDIII antibody titer reached 1:1 024 (Figure 6).

\section{DISCUSSION}

Currently, there is no effective vaccine or antiviral drug to protect against dengue. Passive or active immunizations directed against a variety of DENV proteins are currently being investigated as a potential therapeutic approach for treating DENV infectious diseases. It has been found that the E protein can serve as an effective subunit vaccine candidate due to its capacity to interact with the surface receptors of the host cell and its ability to elicit protective antibodies against virus infection. Both ED II and EDIII have been suggested to be important for binding to the cellular receptor. . $^{40}$

The high antagonistic effect of recombinant West Nile virus (WNV) DIII protein on WNV infection in both mammalian and mosquito cells has strongly suggested that the WNVE DIII protein functions as the receptor-binding domain and is responsible for the recognition and attachment to the cellular receptor. ${ }^{20}$

Virus entry inhibitors also offer an attractive choice for antiviral therapeutics. In fact, this class of inhibitors overcomes some of the problems associated with classical protease inhibitors and DNA or RNA polymerases, such as cellular toxicity and emergence of drug resistance. Virus entry inhibitors have been developed and approved as antiviral agents for the treatment of HIV-1 infections, and one of the most effective ways of preventing DENV infection is by blocking virus entry into target cells. ${ }^{21-23}$ This can be achieved either by blocking the cellular receptors with recombinant DENV DIII envelope proteins or by eliciting neutralizing antibodies that will bind to the virus receptor-binding domains, thereby preventing the virus from binding to the cellular receptors.$^{21-23}$ Recombinant $\mathrm{E}$ protein has been expressed in different systems. ${ }^{24-26}$ The prokaryotic expression system of $E$. coli is widely used due to its rapid culture rate and low cost. However, due to codon usage, certain genes may not be expressed in E. coli. ${ }^{27}$ Rare codons of $\mathrm{E}$ protein-coding mRNA sequence were analyzed by http://nihserver.mbi.ucla.edu/RACC/, and ATA and AGA were found to be continuously distributed on 4-9 sites on its ammonia; CTA, AGG, and ATA were found to be continuously distributed on 56-61 sites; and AGG, CTA, and CCC were continuously distributed on 286-288 sites. In this study, sitedirected mutagenesis was used to identify the synonymous substitutions AGG and AGA, which are the rare codons of $\mathrm{E}$ protein and were not expressed in the full-length gene fragment in E. coli.

We speculated that rare codons may be continuously distributed in the E protein mRNA sequence, and affect its expression in E. coli. As an alternative strategy, the truncated E protein, which maintains its main biological function, can be easily expressed due to its small molecular weight. We truncated the envelope glycoprotein gene, and the truncated EDIII gene was expressed in E. coli cells. This can effectively avoid the continuous existence of thin codons at the N-terminus, the gene fused thioredoxin carried six histidine tags to facilitate recombinant protein purified, increase the recombination solubility and biological activity of protein production.

We used the temperature of $25^{\circ} \mathrm{C}$ to obtain the maximum soluble recombinant protein expression in bacteria while simultaneously reducing the growth rate of $E$. coli to minimize the continuous impact of the presence of these rare codons. Results of the SDSPAGE analysis of the recombinant proteins revealed 
that the expression of the soluble protein accounted for $68.87 \%$ of the recombinant bacterial fusion protein present in the supernatant. A high amount of the natural protein was obtained by using Ni-NTA affinity chromatography. The purity of the resultant protein was $77.86 \%$.

After verification of its biological function, the purified EDIII protein was injected into rabbits to evaluate the induction of neutralizing antibodies. An antibody titer of 1:12 800 was obtained by ELISA with the DENV-2 EDIII protein as a capture antigen. The obtained antisera were used in neutralizing assays on BHK-21 cell lines to determine whether these antibodies could recognize and bind to DENV-2 virus. The polyclonal monospecific antibody was capable of completely neutralizing the infectivity of DENV-2. The DENV-2 EDIII antibodies neutralized up to $80 \%$ of DENV-2 EDIII at a dilution of 1:1 024 on $\mathrm{C} 6 / 36$ cell lines. DIII was initially postulated to be the receptor-binding site for virus particles because of its exposed location at the virus surface. In addition, the presence of the tripeptide motif Arg-Gly-Asp in the loop connecting strands $\beta 6$ and $\beta 4$ in the E proteins of several mosquito-borne flaviviruses suggested that this motif could bind to the integrin family of cell surface matrix receptors. However, this hypothesis was not supported by mutagenesis studies of the yellow fever (YF) virus, and the cellular receptors of flaviviruses have not yet been unequivocally identified. Indeed, a range of different surface molecules could act as flavivirus receptors on different cell types.

In conclusion, a high concentration of DENV-2 EDIII was successfully expressed in E. coli cells. Furthermore, the purified recombinant protein could induce neutralizing antibodies in rabbit antisera, which possessed high neutralization effects on DENV-2 virus. Therefore, the recombinant DENV-2 EDIII protein alone as a subunit vaccine would be more effective than the previous fusion-containing DENV-2 EDIII proteins. The expression and purification strategy may be valuable for developing an inexpensive vaccine against the dengue virus. The identification of the receptor-binding domain of DENV-2 envelope protein can serve as a potential target for the design of antiviral agent development to eradicate this emerging flavivirus infection.

\section{ACKNOWLEDGEMENTS}

This study was supported by grants from the National Natural Science Foundation of China (30872198; 30972566).

\section{REFERENCES}

1. Centers for Disease Control and Prevention (CDC), Division of Vectorborne Infectious Diseasee Dengue Fever. $<$ http://www. cdc.gov/ncidod/ dvbid/dengue/> (2005).

2. Yauch LE, Shresta S. Mouse models of dengue virus infection and disease. Antiviral Res 2008; 80:87-93.

3. Kroeger A, Nathan M, Hombach J. World Health Organization TDR Reference Group on Dengue. World Health Organization TDR Reference Group on Dengue. Nat Rev Microbiol 2004; 2(5):360-361.

4. Perera R, Khaliq M, Kuhn RJ. Closing the door on flaviviruses: Entry as a target for antiviral drug design. Antiviral Research 2008; 80:11-22.

5. Chu JJ, Rajamanonmani R, Li J, Bhuvanakantham R, Lescar $\mathrm{J}, \mathrm{Ng} \mathrm{ML}$. Inhibition of West Nile virus entry by using a recombinant domain III from the envelope glycoprotein. J Gen Virol 2005;86:405-412.

6. Hung JJ, Hsieh MT, Young MJ, Kao CL, King CC, Chang W. An external loop region of domain III of dengue virus type 2 envelope protein is involved in serotype-specific binding to mosquito but not mammalian cells. J Virol 2004; 78:378-388.

7. Crill WD, Roehrig JT. Monoclonal antibodies that bind to domain III of dengue virus E glycoprotein are the most efficient blockers of virus adsorption to Vero cells. J Virol 2001; 75:7769-7773.

8. Hung JJ, Hsieh MT, Young MJ, Kao CL, King CC, Chang W. An external loop region of domain III of dengue virus type 2 envelope protein is involved in serotype specific binding to mosquito but not mammalian cells. J Virol 2004; 78:378-388.

9. Crill WD, Roehrig RT. Monoclonal antibodies that bind to domain III of dengue virus E glycoprotein are the most efficient blockers of virus adsorption to Vero cells. J Virol 2001;75:7769-7773

10. Bhardwaj S, Holbrook M, Shope RE, Barrett ADT, Watowich SJ. Biophysical characterization and vector-specific antagonist activity of domain III of the tick-borne flavivirus envelope protein. J Virol 2001; 75:4002-4007.

11. Chen Y, Maguire T, Hileman RE, Fromm JR, Esko JD, Linhardt RJ. Dengue virus infectivity depends on envelope protein binding to target cell heparin sulfate. Nat Med 1997; 3:866-871.

12. Modis Y, Ogata S, Clements D. Variable surface epitopes in the crystal structure of dengue virus type 3 envelope glycoprotein. J Virol 2005; 79:1223-1231.

13. Modis Y, Ogata S, Clements D. A ligand-binding pocket in the dengue virus envelope glycoprotein. Proc Natl Acad Sci USA 2003; 100:6986-6991.

14. Rey FA, Heinz FX, Mandl C, Kunz C, Harrison SC. The envelope glycoprotein from tick-borne encephalitis virus at $2 \mathrm{~A}$ resolution. Nature 1995; 375:291-298.

15. Wu K, Wu C, Tsao Y, Kuo T, Lou Y, Lin C. Structural basis of a flavivirus recognized by its neutralizing antibody: solution structure of the domain III of the Japanese encephalitis virus envelope protein. J Biol Chem 2003; 278:46007-46013.

16. Modis Y, Ogata S, Clements D, Harrison S. A ligand-binding pocket in the dengue virus envelope glycoprotein. Proc Natl Acad Sci USA 2003; 100:6986-6991. 
17. Crill W, Roehrig J. Monoclonal antibodies that bind to domain III of dengue virus E glycoprotein are the most efficient blockers of virus adsorption to Vero cells. J Virol 2001; 75:7769-7773.

18. Beasley D, Aaskov J. Epitopes on the dengue 1 virus envelope protein recognized by neutralizing $\operatorname{IgM}$ monoclonal antibodies. Virology 2001; 279:447-458.

19. Beasley D, Barrett A. Identification of neutralizing epitopes within structural domain III of the West Nile virus envelope protein. J Virol 2002; 76:13097-13100.

20. Volk D, Beasley D, Kallick D, Holbrook M, Barrett A, Gorenstein D. Solution structure and antibody binding studies of the envelope protein domain III from the New York strain of West Nile virus. J Biol Chem 2004; 279:38755-38761.

21. Chin JFL, Chu JJH, Ng ML. The envelope glycoprotein domain III of dengue virus serotypes 1 and 2 inhibit virus entry. Microbes Infection 2007; 9:1-6.

22. Chu JJH, Rajamanonmani R, Li J, Bhuvanakantham R, Lescar $\mathrm{J}, \mathrm{Ng} \mathrm{ML}$. Inhibition of West Nile virus entry by using a recombinant domain III from the envelope glycoprotein. J Gen
Virol 2005; 86:405-412.

23. Chávez JH, Silva JR, Amarilla AA, Figueiredo LTM. Domain III peptides from flavivirus envelope protein are useful antigens for serologic diagnosis and targets for immunization. Biologicals 2010; 38:613-618.

24. Sugrue RJ, Cui T, Xu J. The production of recombinant dengue virus E protein using Escherichia coli and Pichia pastoris, J Virol Methods 1997; 69:159-169.

25. Chiu MW, Yang YL. Blocking the dengue virus 2 infections on BHK- 21 cells with purified recombinant dengue virus $2 \mathrm{E}$ protein expressed in Escherichia coli. Biochem Biophys Res Commun 2003; 309:672-678.

26. Staropoli I, Clement JM, Frenkiel MP, Hofnung M, Deubel V. Dengue virus envelope glycoprotein can be secreted from insect cells as a fusion with maltose-binding protein. J Virol Methods 1996; 56:179-189.

27. Gustafsson C, Govindarajan S, Minshull J. Codon bias and heterologous protein expression. Trends Biotechnol 2004; 22:346-353. 